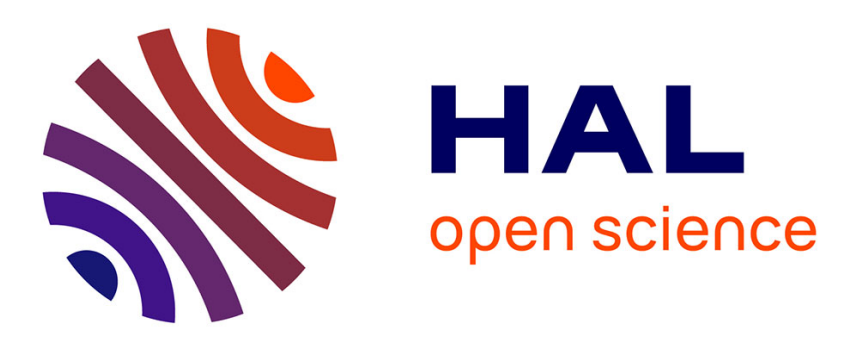

\title{
A New Cyclostationarity-Based Blind Approach for Motor Unit's Firing Rate Automated Detection in Electromyographic Signals
}

Julien Roussel, Michel Haritopoulos, Philippe Ravier, Olivier Buttelli

\section{- To cite this version:}

Julien Roussel, Michel Haritopoulos, Philippe Ravier, Olivier Buttelli. A New Cyclostationarity-Based Blind Approach for Motor Unit's Firing Rate Automated Detection in Electromyographic Signals. BHI 2014 - International Conference of Biomedical and Health Informatics, Jun 2014, Valencia, Spain. pp.4 Pages. hal-01003843

\section{HAL Id: hal-01003843 https://hal.science/hal-01003843}

Submitted on 10 Jul 2014

HAL is a multi-disciplinary open access archive for the deposit and dissemination of scientific research documents, whether they are published or not. The documents may come from teaching and research institutions in France or abroad, or from public or private research centers.
L'archive ouverte pluridisciplinaire HAL, est destinée au dépôt et à la diffusion de documents scientifiques de niveau recherche, publiés ou non, émanant des établissements d'enseignement et de recherche français ou étrangers, des laboratoires publics ou privés. 


\title{
A New Cyclostationarity-Based Blind Approach for Motor Unit's Firing Rate Automated Detection in Electromyographic Signals
}

\author{
Julien Roussel ${ }^{1}$ and Michel Haritopoulos ${ }^{1}$ and Philippe Ravier $^{2}$ and Olivier Buttelli ${ }^{2}$
}

\begin{abstract}
This work focuses on electromyographic (EMG) signal processing. We propose a new blind approach that aims at detecting the firing rates of the activated motor units. The proposed method is based on the fact that, EMGs can be modelled as second-order cyclostationary signals. After application of a Blind Source Separation (BSS) algorithm, we compute a cyclostationarity measure which is the Cyclic Spectral Density (CSD), and we show how one can use it to group the estimated components into independent subspaces and in an automated manner. The proposed classification procedure is based on the concept of subspace BSS techniques, like the Multidimensional Independent Component Analysis (MICA), the difference being that our method allows automatic classification of the estimated source signals. After discarding the subspace corresponding to the noise and computation of a modified CSD measure, the proposed procedure yields to the detection of specific cyclic frequencies corresponding to the discharge frequencies of the Motor Units Action Potential Trains (MUAPTs). Early results obtained from experiments on synthetic EMGs are presented in the paper and research perspectives conclude this work.
\end{abstract}

\section{INTRODUCTION}

Research on bioelectric signals is very active over these last twenty years and, in the meantime, new signal processing techniques emerged, offering new challenges. In particular, Blind Source Separation (BSS) [1] has been applied, e.g. for single muscle activity detection of surface electromyographic signals [2], but also, for the estimation of the foetus' heartbeat rate and subspace dimension [3]. One of the most challenging applications of this technique concerns the exploration of muscle's electrical activity.

A muscle is composed of Muscle Fibres (MFs) organized into Motor Units (MUs). This functional unit is composed of an alpha-motoneuron ( $\alpha$-MN), innervating several MFs (from tens to hundreds MFs depending on the MU and on the muscle characteristics).

Activation of the MU produces an electric field generated by each MF into the MU. The summation of these electric fields provides a specific waveform called Motor Unit Action Potential (MUAP). This full electrical activity is called electromyogram (EMG) and can be recorded using electrodes located on the skin surface (sEMG). When this EMG is composed of several MUs activities it becomes difficult to distinguish the individual MUAPs.

${ }^{1}$ J. Roussel and M. Haritopoulos are with the Univ. Orléans, ENSI de Bourges, PRISME, EA 4229, F28000, Chartres, France \{Julien.Roussel, Michel.Haritopoulos\} at univ-orleans.fr

${ }^{2}$ P. Ravier and O. Buttelli are with the Univ. Orléans, ENSI de Bourges, PRISME, EA 4229, F45072, Orléans, France \{Philippe.Ravier, Olivier.Buttelli\} at univ-orleans.fr
The contraction of MFs requires a train of action potentials which induces a MUAP Train (MUAPT). This train produces a frequency discharge called Firing Rage (FR). In many cases, the FR evaluation of individual MUs is important to understand how the Central Nervous System (CNS) controls the MU and in the case of clinical application it provides information about neuromuscular disorder.

The paper is organised as follows. First, we present the cyclostationary nature of EMG signals. Next, is introduced the model we used to generate synthetic EMGs and the overall concept of BSS techniques; a particular focus is made on Multidimensional Independent Component Analysis (MICA). Follows a detailed presentation of the proposed automated estimation method and the results obtained after its application to simulated EMGs. A discussion concludes this work.

\section{CYCLOSTATIONARY PROPERTIES OF MUSCLE ACTIVITY}

Even under isometric conditions and in constant force, the MUAPT is not strictly periodic and has a mean Inter-Spike Interval (ISI) equal to the inverse of the FR. Instantaneous ISI is not fully constant over time; it exhibits a random variation called jitter [4]. Finally, a MUAPT writes:

$$
y(t)=\sum_{i \in \mathbb{N}} x\left(t-\frac{i}{F R}+\tau_{i}\right)
$$

where $x$ stands for the MUAP waveform and $\tau_{i}$ denotes the jitter.

This signal exhibits a hidden periodicity at the FR, called cyclostationarity. It has been shown [5] the considered model in (1) is a second-order cyclostationary signal (CS) that leads to a periodic Instantaneous Autocorrelation Function (IAF):

$$
\Gamma_{x x}(t, \tau)=\mathbb{E}\left[x\left(t-\frac{\tau}{2}\right) \overline{x\left(t+\frac{\tau}{2}\right)}\right]
$$

The two-dimensional Fourier transform of $\Gamma_{x x}(t, \tau)$ along $t$ and $\tau$ provides the cyclic spectrum of the signal of interest, with $\alpha$ the cyclic frequency and $f$ the spectral frequency, associated to $t$ and $\tau$, respectively. That leads to the Cyclic Spectrum Density (CSD) hereafter:

$$
\begin{aligned}
S_{x x}(\alpha, f) & =\iint_{\mathbb{R}^{2}} \Gamma_{x x}(t, \tau) e^{-2 i \pi(t \alpha+\tau f)} d t d \tau \\
& =\mathbb{E}\left[X\left(f-\frac{\alpha}{2}\right) \overline{X\left(f+\frac{\alpha}{2}\right)}\right]
\end{aligned}
$$

where $x$ stands for the MUAP waveform and $X$ denotes its Fourier transform. Considering a second-order cyclostationary signal, the periodicity in time of the IAF generates 
spectral lines along values of the cyclic frequency $\alpha$. As we will demonstrate in what comes next, the CSD proves to be a useful tool in order to reveal hidden periodicities present in the MUAPT.

\section{EMG SIGNAL PROCESSING}

\section{A. EMG Modelling}

For the simulated EMG signals and the experiments present in this paper, we used (1) to generate each MUAP. Summation of the action potentials generated by the fibers corresponding to these MUAPs, leads to the following synthetic EMG model:

$$
s_{E M G}(t)=\sum_{i=1}^{N} y_{i}(t)+\nu(t)
$$

where $N$ and $i \in[1, N]$ denote the number of active MUs and the MUAP index, respectively, whereas $\nu(t)$ stands for an iid Gaussian noise. The MUAPT $y$ is computed following (1) and its jitter $\tau_{i}$ is drawn from a normal distribution with zero mean and $\sigma_{i}^{2}$ variance. According to Clamann's law [4], standard deviation is computed following $\sigma_{i}=$ $\left(0.91 / F R_{i}^{2}\right)+4 \times 10^{-3}, \quad i \in[1, N]$. Finally, the simulated MUAPTs use a library template of synthetic MUAP waveforms based on model (4) (interested readers are referred to [6] for details).

\section{B. Blind Source Separation}

Given a set of $M$ observed signals $x_{i}(t), i \in[1, M]$, BSS methods aim to extract the $N$ unknown source signals $s_{j}(t), j \in[1, N]$, with $N \leq M$, which are drowned in the observations contaminated by additive noise $n(t)$ :

$$
\mathbf{x}(t)=A \mathbf{s}(t)+n(t)
$$

where $\mathbf{x}(t)=\left[x_{1}(t), x_{2}(t), \ldots, x_{M}(t)\right]^{\dagger}$ and $\mathbf{s}(t)=\left[s_{1}(t), s_{2}(t), \ldots, s_{N}(t)\right]^{\dagger}$ denote the observations (also called mixtures), and the unknown source vector, respectively, with $\dagger$ the transpose operator and with $A$ an unknown $M \times N$ full rank mixing matrix. Various methods for estimating the unmixing matrix depending on the conditioning of $A$ and the number of source as well mixture vectors exist in the literature; one can compute the pseudo-inverse of $A$ or use adaptive filters which avoid matrix inversion [7]. The model in (5) is known in the literature as the noisy linear instantaneous model and assumes statistical independence of the unknown source signals and at most one Gaussian source vector component. Regarding the noise, is generally assumed to follow a normal distribution.

\section{Multidimensional Independent Component Analysis}

The Multidimensional Independent Component Analysis (MICA) [8] consists of a linear separation of mixed signals into independent groups instead of solely single independent components (ICs). Its author applied this method to real foetal electrocardiogram (FECG) data. From a set of observations recorded with skin electrodes placed in the expectant mother's abdominal and thoracic areas, he first applies the
BSS algorithm JADE [9] to a three-dimensional set of these recordings to extract an equal number of independent components. Then, based on a simple visual inspection, the author groups two of them into a two-dimensional subspace corresponding to the maternal ECG (MECG); we will show in IV-C how to automate this classification step using the cyclostationary properties of the signal of interest.

\section{SIMULATED DATA AND METHOD}

\section{A. Generating Synthetic EMG Mixtures}

For our experiments, we needed to construct a simulated EMG mixture vector. For this, we used (4) described in section III-A. Our surface EMG acquisition test bench consisting of seven equidistant surface electrodes, we took into account the slight delay with which each MUAPT arrives from the first towards the last sensor. Once the MUAPT delayed versions computed, they are mixed up with a real well conditioned (i.e. non singular) mixing matrix $A_{E M G}$. Finally, a Gaussian noise $g_{n}(t)$ is added to each one of the input channels. Hence, the following model corresponds to the BSS noisy linear instantaneous one in (5):

$$
\mathbf{x}_{\mathrm{EMG}}(t)=A_{E M G} \mathbf{s}_{\mathrm{EMG}}(t)+g_{n}(t)
$$

where $\mathbf{s}_{\mathrm{EMG}}(t)$ is the vectorial form of the unknown EMG signals in (4).

The variables in our simulation procedure are:

- the number $N$ of the activated MUs,

- the firing rates for each one of the activated MUs (in $H z)$,

- the size $M$ of the observations vector $\mathbf{x}_{\mathrm{EMG}}$,

- the mixing matrix $A_{E M G}$,

- the jitter level as rate of the Clamann's law $\sigma_{i}$ in $\%$, and,

- the $S N R$ expressed in $d B$ (amount of noise $g_{n}$ ).

In the following and in order to clarify the presentation of the simulation results, the above variables are assigned the next values: $N=4$ with respective firing rates $F R_{i}=$ $\{10.8,11,13.5,13.8\} H z, M=7, A_{E M G}$ is a $M \times N$ mixing matrix containing random values drawn from the normal distribution, jitter level in $\{10,50,100\} \% \sigma_{i}$ and $S N R$ in $\{-15,-10,-5\} d B$. For comparison purposes, the same mixing matrix $A_{E M G}$ and additive noise vector $g_{n}(t)$ have been used in all simulations.

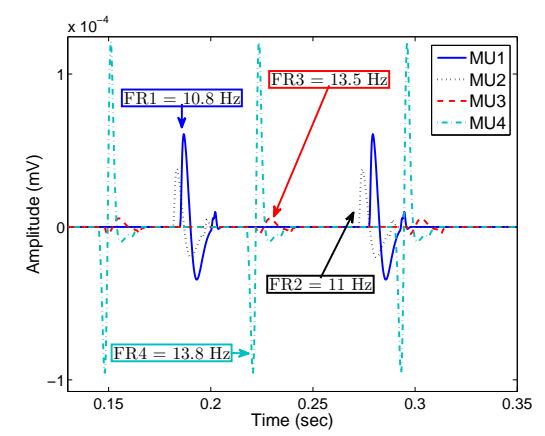

Fig. 1. The 4 MUAP library templates used for the simulations. 


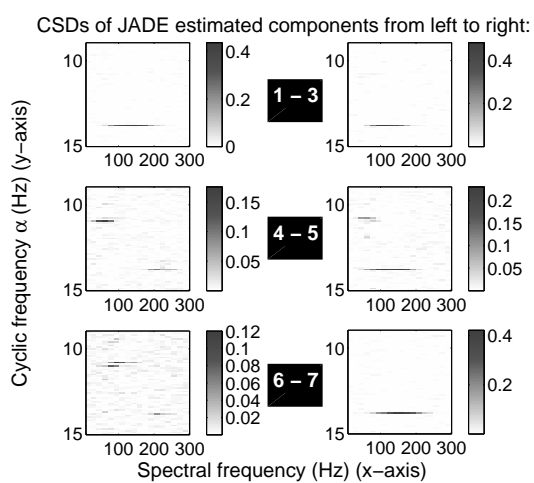

Fig. 2. CSDs of 6 out of 7 estimated ICs using the JADE algorithm.

\section{B. Why Use Cyclostationary Measures?}

Fig. 1 shows the time waveform of the raw MUAP library templates used in all simulations for this work. One can easily remark that, their respective shapes are all different (but, in general, they may also have some similarities), their amplitudes have a large dynamic range and, also, they may overlap in time. This is some of the major complexities that make decomposition or firing rates estimation tasks more difficult [10].

Once the mixing vector computed according (6), combinations of the aforementioned complexities together with the strength of additive Gaussian noise of the mixing model and the amount of jitter, make EMG signal processing an even more challenging task. It is very hard to distinguish the individual MUAP shapes from the time plots of the $\mathbf{x}_{\text {EMG }}$ mixture vector's individual components. Hence, we used envelope spectrum analysis of the observations and we compared the obtained results concerning FR estimation, with those obtained with the proposed method based on the intrinsic cyclostationary properties of EMG signals (envelope spectrum is issued from the Fourier transformed squared analytical signal of interest). As cyclostationarity measure, we used the integrated CSD (iCSD) obtained by summing over all the spectral frequencies $f$ for every cyclic frequency $\alpha$.

\section{The Procedure for the Firing Rates detection}

The following steps compose the proposed MU's firing rate automated detection procedure:

1) Apply JADE to the mixing vector $\mathbf{x}_{\mathrm{EMG}}(t)$ and obtain estimated independent components $J A D E_{i}, i \in$ $[1, M]$.

2) Compute the CSD and then the iCSD for each extracted independent component previously computed.

3) Compute and compare standard deviation and kurtosis values of the CSDs and put into an independent subspace those corresponding to the noise.

4) For each iCSD computed in step 2, except for those corresponding to the noise subspace (step 3), subtract its maximum value. If the new vector exhibits null values, then, the ICs whose null values correspond to the same cyclic frequency $\alpha$ span a subspace of signal components corresponding to a MUAP firing at that cyclic frequency.

The above proposed procedure employs one of the most widely used BBS algorithms (JADE) which is based on fourth-order statistics. Other BSS algorithms may be used for the separation task (step 1).

\section{RESULTS}

For the simulations, the variables are given the values detailed in section IV-A. In real world EMG signals, the number of active distinguishable MUs - even for minimum Maximum Voluntary Contraction (MVC) rates, is at least equal to several hundreds. But, for the sake of clarity and to test the feasibility of the proposed method, we have chosen $N=4$.

\section{A. A Concrete Case Simulation Example}

Let us now illustrate the application of the proposed FR detection procedure by considering a simulated EMG mixture with a $100 \% \sigma_{i}$ jitter and $-10 d B S N R$. Envelope spectrum analysis of the $\mathbf{x}_{\mathrm{EMG}}(t)$ vector, reveals 2 among the 4 unknown, a priori, firing rates. Envelope spectrum analysis of the estimated ICs after application of the JADE algorithm, does not detect any additional FR. After applying the proposed procedure, one obtains the CSDs of the JADEseparated ICs. Now, and in order to decide whether an IC should fit the noise subspace, one computes the standard deviation and kurtosis $\left(\kappa_{i}, 1 \leq i \leq M\right)$ values of the obtained CSDs. Obtained results are summarised in Table I.

The IC corresponding to the noise subspace is the second one, not only because its CSD's standard deviation value is the smallest one, but also, because of its CSD's low kurtosis value comparatively to those of the remaining ICs, which indicates a very noisy CSD plot. The CSDs of the remaining six ICs are plotted in Fig. 2; one can easily remark highvalued spectral lines at cyclic frequencies equal to $10.8 \mathrm{~Hz}$, $11 \mathrm{~Hz}$ and $13.8 \mathrm{~Hz}$. Thus, the subspace containing useful information about the signal of interest is spanned by six components while the noise subspace is one-dimensional.

The detection of the previously detected firing rates is automated by computing the integrated CSDs of the sixdimensional subspace and then subtracting from each one of them its maximum value. Therefore, one obtains the plot of Fig. 3. The modified iCSDs of components $1,3,5,7$ exhibit a single null value at cyclic frequency $\alpha=13.8 \mathrm{~Hz}$, the one of component 4 has a null at $\alpha=11 \mathrm{~Hz}$ and, finally, the iCSD of component 6 vanishes at $\alpha=10.8 \mathrm{~Hz}$. These cyclic frequencies correspond to firing rates $F R_{1,2,4}$ (section

TABLE I

STANDARD DEVIATION $\left(\sigma_{C S D_{i}}\right)$ AND KURTOSIS $(\kappa)$ VALUES OF THE $i^{t h}$ INDEPENDENT COMPONENT'S CSD.

\begin{tabular}{|c|c|c|c|c|c|c|c|}
\hline$I C_{i}$ & 1 & 2 & 3 & 4 & 5 & 6 & 7 \\
\hline$\sigma_{C S D_{i}}$ & 0.36 & 0.02 & 0.34 & 0.07 & 0.19 & 0.05 & 0.33 \\
\hline$\kappa_{i}$ & 55.7 & 7.4 & 55.8 & 28.5 & 52 & 21.1 & 55.7 \\
\hline
\end{tabular}




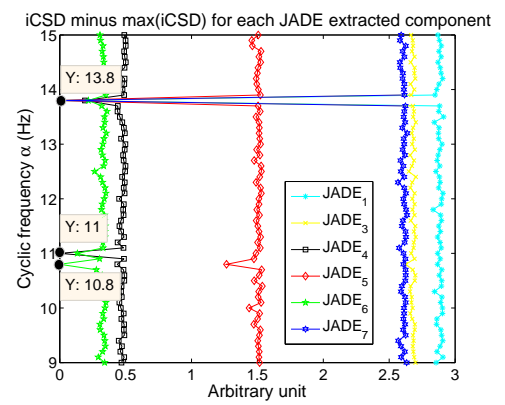

Fig. 3. Modified iCSD of all JADE-estimated ICs except the $2^{\text {nd }}$ one corresponding to the noise subspace.

IV-A). Only the $F R_{3}=13.5 \mathrm{~Hz}$ still remained undetectable most likely due to the relatively small amplitude of the third MUAP (Fig. 1).

\section{B. Synthesis of the Obtained Results}

Many different synthetic EMG mixtures have been tested. Table II resumes the obtained results with the variables' values given in section IV-A.

For each pair $\{S N R$ (rows), jitter (columns) $\}$, the columns $\mathrm{A}, \mathrm{B}$ and $\mathrm{C}$ of the table show the number of detected firing frequencies associated to the active MUs. The first two columns, $\mathrm{A}$ and $\mathrm{B}$, correspond to the results given after visual inspection of the envelope spectrum of the raw mixtures, and the JADE-separated independent components, respectively, whereas, the third column $\mathrm{C}$, gives the results after application of the procedure described earlier in section IV-C.

With $100 \% \sigma_{i}$ jitter values and for $S N R$ ratios smaller than $-5 d B$, the proposed method achieves detection of all firing rates, except the one at $13,5 d B$. In the worst case, it detects as much firing frequencies as with an envelope spectrum analysis after ICA separation of the raw data. The fact that the proposed method did not succeed to detect the remaining FR, is mainly due to the very low amplitude range of the associated MU with respect to these of the remaining MUs (dashed red curve of Fig. 1).

\section{DISCUSSION}

In the last decade, various techniques for EMG decomposition have been proposed in the literature (e.g. [11]). The work we present here is a novel method for estimating the number of MUs and their firing rates in surface EMGs. It takes advantage of the intrinsic cyclostationary properties of

TABLE II

NUMBER OF DETECTED MOTOR UNITS

\begin{tabular}{|c||c|c|c||c|c|c||c|c|c|}
\cline { 2 - 11 } \multicolumn{1}{c|}{} & \multicolumn{10}{c||}{ Jitter $\left(\% \sigma_{i}\right)$} \\
\cline { 2 - 11 } \multicolumn{1}{c|}{} & \multicolumn{3}{c|}{10} & \multicolumn{3}{c||}{50} & \multicolumn{3}{c|}{100} \\
\hline SNR & $\mathrm{A}$ & $\mathrm{B}$ & $\mathrm{C}$ & $\mathrm{A}$ & $\mathrm{B}$ & $\mathrm{C}$ & $\mathrm{A}$ & $\mathrm{B}$ & $\mathrm{C}$ \\
\hline$-5 d B$ & 2 & 3 & 3 & 3 & 3 & 3 & 3 & 3 & 3 \\
\hline$-10 d B$ & 2 & 2 & $\mathbf{3}$ & 2 & 2 & $\mathbf{3}$ & 2 & 2 & $\mathbf{3}$ \\
\hline$-15 d B$ & 1 & 2 & $\mathbf{3}$ & 1 & 2 & $\mathbf{3}$ & 1 & 1 & $\mathbf{3}$ \\
\hline
\end{tabular}

such signals, which, at the best of our knowledge, has not been reported yet in the dedicated literature, and it introduces a new automated classification step of the BSS estimated components into independent subspaces. The obtained results after application of the proposed method to synthetic EMGs are very promising.

With the proposed method and under certain conditions one obtains a gain over traditional frequency domain signal processing techniques. Future research should focus on the validation of the proposed method's estimation accuracy and of its robustness against different BSS algorithms, when applied to simulated EMG mixtures, with more or less closely spaced FRs, with different numbers and various relative amplitude range for the MUs used for the simulated mixtures. Current research in that direction is ongoing. Also, its extension and application on real world EMG datasets is a very challenging task due to the important number of active MUs which can vary between ten to more than several thousands depending on the muscle.

\section{ACKNOWLEDGMENT}

Authors would like to thank Prof. Dr. Dario Farina, Director of the Department of Neurorehabilitation Engineering at the University Medical Center of Goettingen, Germany, for having kindly provided us code for action potential simulation.

\section{REFERENCES}

[1] C. Jutten and J. Herault, Blind separation of sources, part I: An adaptive algorithm based on neuromimetic architecture, Signal Processing, vol. 24(1), 1991, pp. 1-10.

[2] D. Farina, Blind Separation of Linear Instantaneous Mixtures of Nonstationary Surface Myoelectric Signals, IEEE Transactions On Biomedical Engineering, vol. 51, 2004, pp. 1555-1567.

[3] M. Haritopoulos, J. Roussel, C. Capdessus and A.K. Nandi, Cyclostationarity-Based Estimation of the Foetus Subspace Dimension from ECG Recordings, in Proc. of XIII Mediterranean Conference on Medical and Biological Engineering and Computing, MEDICON 2013, IFMBE Proceedings, vol. 41, Laura M. Roa Romero eds., Springer, 2014, pp. 726-729.

[4] H.P. Clamann, Statistical Analysis of Motor Unit Firing Patterns in a Human Skeletal Muscle, Biophysical journal, vol. 9, 1969, pp. 12331251.

[5] J. Roussel, M. Haritopoulos, P. Ravier and O. Buttelli, Cyclostationary Analysis of Electromyographic Signals, in Proc. of $21^{\text {st }}$ European Signal Processing Conference, EUSIPCO 2013, Marrakech, Morocco, Sept. $9^{t h}-13^{t h}, 2013$.

[6] D. Farina, L. Mesin, S. Martina and R. Merletti, A Surface EMG Generation Model With Multilayer Cylindrical Description of the Volume Conductor, IEEE Transactions on Biomedical Engineering, vol. 51(3), 2004, pp. 415-426.

[7] I. Sahin, N. Yilmazer, M. A. Simaan, A Method for Subsample Fetal Heart Rate Estimation Under Noisy Conditions, IEEE Transactions on Biomedical Engineering, vol. 57(4), 2010, pp. 875-883.

[8] J.F. Cardoso, Multidimensional Independent Component Analysis, in Proc. of the 1998 IEEE International Conference on Acoustics, Speech and Signal Processing, vol. 4, 1998, pp. 1941-1944.

[9] J.F. Cardoso and A Souloumiac, Blind beamforming for non Gaussian signals, Radar and Signal Processing, IEE Proceedings F, vol. 140(6), Dec. 1993, pp. 362-370.

[10] C.J. De Luca, A. Adam, R. Wotiz, L.D. Gilmore and S.H. Nawab, Decomposition of Surface EMG Signals, Journal of Neurophysiology, vol. 96, 2006, pp. 1646-1657.

[11] V. Glaser, A. Holobar, D. Zazula, Real-Time Motor Unit Identification From High-Density Surface EMG, IEEE Transactions on Neural Systems and Rehabilitation Engineering, vol. 21(6), 2013, pp. 949958. 